\title{
PROPOSTA DE INSTRUMENTO PARA COLETA DE DADOS DE ENFERMAGEM EM UMA UNIDADE DE TERAPIA INTENSIVA FUNDAMENTADO EM HORTA*
}

\author{
PROPOSAL OF INSTRUMENT FOR COLLECTION OF DATA OF NURSING IN A UNIT OF INTENSIVE \\ THERAPY BASED ON HORTA*.
}

\section{PROPUESTA DE INSTRUMENTO PARA COLECTA DE DATOS DE ENFERMERÍA EN UNA UNIDAD DE TERAPIA INTENSIVA FUNDAMENTADO EN HORTA*}

\section{Luciano Ramos de Lima ${ }^{1}$, Marina Morato Stival ${ }^{2}$, Luciene Ramos de Lima ${ }^{3}$, Cleydson Rodrigues de Oliveira ${ }^{4}$, Tânia Couto Machado Chianca ${ }^{5}$}

RESUMO: O processo de enfermagem é um método que possibilita o enfermeiro desenvolver e aplicar seus conhecimentos técnico-científicos, evidenciar sua prática profissional e proporcionar uma assistência sistematizada. Objetivou-se elaborar e validar um instrumento de coleta de dados para paciente internado na unidade tratamento intensivo (UTI). O estudo foi dividido em três etapas construção do instrumento fundamentado na Teoria de Necessidades Humanas Básicas (NHB) de Wanda A. Horta, validação de aparência e conteúdo, e refinamento do instrumento. A validação de aparência e conteúdo foi realizada por 10 enfermeiros especialistas. Os avaliadores sugeriram algumas modificações, tanto no conteúdo quanto na aparência, e julgaram que o instrumento poderá facilitar a coleta de dados, a análise, o julgamento e uma proposta de intervenções de enfermagem específicas aos problemas levantados.

PALAVRAS CHAVE: Teoria de Enfermagem; Coleta de Dados; Unidades de Terapia Intensiva.

ABSTRACT: The nursing process is a method that makes it possible to the nurse to develop and apply the technical-scientific knowledge, to evidence his practical professional and to provide a systemized assistance. The purpose of this study is to elaborate and to validate an instrument of collection of data for interned patient in the intensive care unit (UTI). The study was divided in three-stages - construction of the instrument based on Wanda A. Horta's Theory of Basic Human Needs (BHN), validation of content and appearance and refinement of the instrument. The validation of appearance and content was carried through by 10 nurses specialist. The appraisers had suggested some modifications, in the content and also in the appearance, and had judged that the instrument will be able to facilitate the collection of data, the analysis, the judgment and a proposal of specific interventions of nursing to the raised problems.

KEY WORDS: Nursing Theory; Data Collection; Intensive Care Units.

RESUMEN: El proceso de enfermería es un método que posibilita al enfermero desarrollar y aplicar sus conocimientos técnico-científicos, evidenciar su práctica profesional y proporcionar una asistencia sistematizada. Se objetivó elaborar y validar un instrumento de colecta de dados para pacientes ingresados en la unidad tratamiento intensivo (UTI). Es estudio fue dividido en tres etapas- construcción del instrumento fundamentado en la Teoría de Necesidades Humanas Básicas (NHB) de Wanda A. Horta, validación de apariencia y contenido, y enfermeros especialistas. Los evaluadores sugirieron algunas modificaciones, tanto en el contenido como en la apariencia, y juzgaron que el instrumento podrá facilitar la colecta de datos, el análisis, el juzgamiento y una propuesta de intervenciones de enfermería específicas a los problemas levantados.

PALABRAS CLAVE: Teoría de Enfermería; Colecta de Datos; Unidades de Terapia Intensiva.

\footnotetext{
* Trabalho parte de um projeto do Núcleo de estudos e pesquisas sobre a sistematização do cuidar em enfermagem da Escola de Enfermagem da UFMG. Belo Horizonte/MG.

${ }^{1}$ Enfermeiro, Professor da Escola de Enfermagem da UFMG. Pós-graduado em UTI pela UFMG. Belo Horizonte/MG (031) 3424-2718. E-mail: enframosll@gmail.com.

${ }^{2}$ Enfermeira, Mestranda da Escola de Enfermagem da UFMG, Belo Horizonte/MG. E-mail: mstival@gmail.com.

${ }^{3}$ Graduanda de Enfermagem, UniEvangélica Centro Universitário, Belo Horizonte/MG. E-mail: enflucieneramos@gmail.com

${ }^{4}$ Enfermeiro, Pós-graduado em UTI da Escola de Enfermagem da UFMG, Belo Horizonte/MG. E-mail: cleydson.net@bol.com.br

${ }^{5}$ Enfermeira, Professora Doutora da Escola de Enfermagem da UFMG, Belo Horizonte/MG. E-mail: taniacmc@enf.ufmg.br
} 


\section{INTRODUÇÂO}

O objeto da enfermagem é o cuidado humano que deve ser prestado com qualidade. Considera-se importante que enfermeiros desenvolvam o pensamento crítico e sua capacidade de tomar decisões por ser reconhecidamente um agente de transformação das condições de vida, atuando diretamente no processo saúde-doença e no bem estar dos indivíduos, famílias e comunidade. O cuidado de enfermagem deve ser realizado com planejamento e de forma sistematizada, o que pode ocorrer por meio da implementação do processo de enfermagem (PE).

A ação profissional deve ser marcada pela compreensão e reflexão teórica, que caracterizam o pensamento crítico. Ambas são inerentes aos papéis desempenhados pelos enfermeiros na assistência, no ensino, na administração e na pesquisa (BARROS, et al., 2002)

Por volta de 1950 percebe-se as primeiras alusões ao PE que tem sido visto como a aplicação do método científico na prática de enfermagem. Nesse sentido, (DOENGES \& MOORHOUSE, 1999) relatam que há muitos anos, os profissionais de enfermagem identificaram um processo para solução de problemas que combinavam elementos mais desejáveis da arte da enfermagem com os elementos mais relevantes da teoria de sistemas e que pressuponha a utilização do método científico.

No Brasil, a competência do enfermeiro para diagnosticar está regulamentada pela Resolução do Conselho Federal de Enfermagem (COFEN) $n^{\circ}$ 272/2002 que dispõe sobre a instituição da Sistematização da Assistência de Enfermagem (SAE) em instituições de saúde públicas e privadas (BRASIL, 2002).

O PE possui cinco fases: investigação (coleta sistematizada de dados dos pacientes e seus respectivos problemas); diagnósticos de enfermagem (identificação de problemas pela análise dos dados coletados); planejamento (determinação dos resultados desejados, "metas específicas" e identificação das intervenções para alcançar resultados); implementação (colocação do plano em ação) e avaliação (determinação do sucesso no alcance dos resultados e decisão quanto às mudanças a serem feitas) (BETEGHELLI, et al. 2005). As diferentes etapas do PE se inter-relacionam e envolvem um contínuo processo de raciocínio e julgamento clínico que direciona as ações de enfermagem (ALFARO-LEFREVE, 2005).

Assim, alguns autores afirmam que o PE pode ser aplicado em qualquer cenário de cuidado à saúde, utilizando os mais diversos arcabouços teóricos ou conceituais e nos mais variados contextos. O plano de cuidado adequadamente utilizado é capaz de guiar e favorecer a continuidade do cuidado na assistência de enfermagem, por meio da facilitação da comunicação entre enfermeiros e outros profissionais que prestam os cuidados (DOENGES \& MOORHOUSE,1999; LIMA et al, 2006).

Outros autores apontam o PE como uma ferramenta essencial do enfermeiro para a prestação de cuidados. As ações devem ser deliberadas, sistematizadas, ordenadas, individualizadas e eficazes (CARPENITO, 1997; ATKINSON \& MURRAY, 1998; GEORGE et al., 2000; CIANCIARRULO, 2001; BARROS et al., 2002; CARVALHO \& GARCIA, 2002; TIMBY, 2002; ALFARO-LEFREVE, 2005).

Pacientes internados em UTI necessitam de assistência de enfermagem especializada e contínua. Atualmente, estes pacientes recebem cuidados muitas vezes sem planejamento, o que demonstra pouca fundamentação teórica. Na prática clínica temse presenciado que enfermeiros intensivistas em geral, ainda não documentam a assistência que prestam de forma sistematizada. Desta forma, destaca-se a necessidade da organização dos cuidados dispensados a estes pacientes, por meio da implementação do PE fundamentado em uma teoria que atenda as necessidades de pacientes críticos internos na UTI.

Um estudo que utilizou o PE em pacientes inconscientes internados em uma unidade de terapia intensiva de um hospital de Curitiba-PR, fundamentado na Teoria das Necessidades Humanas Básicas e utilizando a Escala de coma de Glasgow para avaliação neurológica, concluiu que a experiência contribuiu para o desenvolvimento de espírito de liderança no enfermeiro e é um método de avaliação profissional. Entretanto, para utilizar o PE o profissional deve ser capacitado e treinado a especificar cuidados adequados a cada paciente, a formular planejamentos específicos, fazer prescrições de enfermagem e evoluções do estado de saúde do paciente (GOMES, et al, 1987).

Uma das críticas constantes dos enfermeiros quanto à coleta de dados se refere ao tempo que esta atividade consome. Na tentativa de desmistificar esta afirmativa um estudo realizado em uma unidade de terapia semi-intensiva no Estado de São Paulo, foi elaborado um instrumento para a coleta de dados e prescrição de enfermagem utilizando um instrumento com questões do tipo "checklist" onde os enfermeiros deveriam assinalar itens necessários à prescrição de enfermagem e os horários adequados. Este instrumento reduziu em $50 \%$ o tempo para elaborar a prescrição de enfermagem (SPERANDIO \& ÉVORA, 2004). Em outro estudo, pesquisadores entrevistaram enfermeiras dos centros de tratamento intensivo do Rio de Janeiro e constataram a ausência de utilização do processo de enfermagem nas unidades. As autoras propuseram o desenvolvimento de um protótipo utilizando a Classificação Internacional da Prática de Enfermagem (CIPE) durante a aplicação 
das etapas do processo de enfermagem diagnósticos, intervenções e resultados de enfermagem- (FULY et al., 2003).

Em um hospital de referência em Fortaleza, Ceará, foram identificados diagnósticos de enfermagem em pacientes vítimas de traumatismos crânio-encefálico e foi elaborado um modelo para a sistematização da assistência de enfermagem utilizando a taxonomia proposta pela North American Nursing Diagnoses Association (NANDA) para a etapa de diagnósticos de enfermagem (FEITOZA, et al., 2004). No estudo foi utilizado o modelo de raciocínio clínico proposto por (RISNER, 1986), para a etapa do PE de formulação dos diagnósticos de enfermagem e demais etapas do PE. Alguns pesquisadores têm apontado que existe uma tendência na enfermagem de utilização de classificações de enfermagem como instrumentos para nortear a prática e que há necessidade de reformulação curricular dos cursos, a partir dos conceitos empregados nas classificações (CHIANCA, et al., 2000).

Aplicar instrumentos de enfermagem validados e socializados no meio científico contribui para a melhoria da qualidade da assistência de enfermagem, bem estar do cliente e familiares, além de contribuir para o conhecimento científico e maior visibilidade do trabalho de enfermagem em equipe multiprofissional de saúde (CARVALHO \& GARCIA, 2002; MC CLOSKEY \& BULECHEK, 2004). Entretanto, considera-se necessário a utilização de referencial teórico que direcione as etapas do PE.

Neste estudo optou-se por utilizar o modelo teórico defendido por Horta (HORTA, 1979) que foi desenvolvido a partir da Teoria de Maslow que se fundamenta nas necessidades humanas básicas (NHB) e no referencial de João Mohana (HORTA, 1975). Este agrupa as necessidades em psicobiológicas, psicossociais e psico-espirituais. Acredita-se que este modelo atenda as necessidades de pacientes críticos internos em UTI.

Tendo em vista que o paciente crítico internado em uma UTI é um ser dependente dos cuidados de enfermagem o enfermeiro deve ser capaz de detectar as necessidades psicobiológicas, psicossociais e psico-espirituais comprometidas, e auxiliá-lo no atendimento das necessidades que estão afetadas, de acordo com o grau de prioridades estabelecido no plano de atendimento de enfermagem. Os enfermeiros devem atuar de forma sistematizada na assistência de enfermagem prestada ao paciente crítico interno em UTI utilizando todas as etapas do PE. Neste sentido a coleta de dados, é a etapa entendida como primordial, onde as informações são coletadas e irão subsidiar o planejamento da assistência.

Para focar essa problemática é que se propôs esta pesquisa, cujo objetivo foi elaborar e validar um instrumento de coleta de dados, primeira etapa da sistematização da assistência de enfermagem para pacientes internos em UTI fundamentado no modelo conceitual das NHB da Teoria de Wanda A.Horta (1979).

\section{METODOLOGIA}

O presente trabalho foi dividido em três etapas, sendo a primeira a construção do instrumento de coleta de dados, a segunda a validação de aparência e conteúdo realizada por enfermeiros especialistas e a terceira o refinamento do instrumento.

Primeira etapa: a construção do instrumento.

Para elaboração do instrumento foi realizado um levantamento bibliográfico sobre o exame físico e cuidados críticos de pacientes em UTI em livros texto. Este levantamento foi fundamento em (HUDAK \& GALLO, 1997; BARROS, et al., 2002; PORTO, 2002; JARVIS, 2002; ANDRIS, 2006).

A teoria selecionada para guiar o $\mathrm{PE}$ que fundamenta o instrumento foi a de NHB de Horta (HORTA, 1979). O instrumento de coleta de dados foi elaborado de forma a contemplar as necessidades psicobiológicas, psicoespirituais e psicossociais. conteúdo.

Segunda etapa: a validação de aparência e

A validação consiste em avaliar se o instrumento mede aquilo que se propõe a medir (LOBIONDO-WOOD \& HABER, 2002; POLIT, et al. 2004). O instrumento foi entregue a dez enfermeiros especialista na área para avaliar o conteúdo e aparência com vistas a validar o instrumento.

Os critérios utilizados para escolha dos juízes foram: enfermeiros que possuíam pós-graduação em UTI (especialização, mestrado e doutorado); e com experiência profissional de no mínimo três anos de prática assistencial em UTI.

Os juízes avaliaram as cinco NHB contempladas no instrumento julgando os principais sinais e sintomas. Neste sentido verificaram se eram aplicáveis na avaliação do paciente crítico em UTI. Utilizou-se uma escala de avaliação onde os especialistas deveriam assinalar se concordavam, discordavam e ainda um espaço destinado a sugestões para o acréscimo, retirada ou modificações nos itens do instrumento.

Terceira etapa: refinamento dos itens do instrumento de coleta de dados.

Os juízes assinaram um termo de consentimento livre e esclarecido para participarem do estudo. Sendo respeitado os preceitos da Resolução do Conselho Nacional de Saúde n. 96, de 10/10/96.

\section{RESULTADOS}

A construção do instrumento (Anexo I) foi o primeiro passo. Neste foi realizado uma revisão das principais teorias de enfermagem (GEORGE, et al., 
2000), sendo selecionada a Teoria das NHB (HORTA, 1979). Optou-se por esta teoria por considerar que esta favorece uma avaliação do paciente como um todo indivisível.

A construção do instrumento obedeceu os critérios do exame físico céfalo-caudal, com o objetivo de identificar e avaliar o paciente em todas as necessidades e de possibilitar a identificação de problemas (BARROS, et al., 2002; PORTO, 2002; JARVIS, 2002; ANDRIS 2006). Na primeira parte do instrumento contava com dados de identificação, da entrevista, história pregressa de doenças e de fatores de riscos. Os itens subseqüentes estavam direcionados para o exame físico e estavam distribuídos em NHB: psicobiológicas (percepção, exame da cabeça e pescoço, oxigenação, circulação, regulação fisiológica, hidratação, nutrição, eliminação, integridade física, higiene, mecânica corporal, sono e repouso), psicossociais (segurança) e psicoespiritual (religião).

Após a confecção do instrumento, foi entregue a dez enfermeiros para avaliação. Destes, 60\% possuíam especialização e $40 \%$ eram assistências com mais de três anos de experiência em UTI.

Os especialistas avaliaram cada item do instrumento distribuídos nas NBH. Quanto aos ítens de identificação, $90 \%$ dos enfermeiros consideraram que o instrumento era extenso por possuir quatro páginas e de difícil aplicação na prática clínica cotidiana. Foi sugerido separar o instrumento em duas partes: a primeira (Anexo, Parte I) para ser preenchida somente na admissão com os dados referentes à identificação e entrevista e a segunda (Anexo, Parte II) para ser evoluída diariamente, de forma a ocupar no máximo frente e verso de uma folha e assim ter maior aplicabilidade na UTI. Neste sentido, $50 \%$ dos juízes sugeriram acrescentar horário de admissão, procedência e um campo destinado a cirurgias prévias.

A avaliação das NHB psicobiológicas foi uma das partes onde houve mais discordâncias e sugestões de alterações. Além disso, essa avaliação é ainda pouco realizada no cotidiano dos enfermeiros e a colaboração dos juízes com sugestões advindas da experiência prática e teórica deles foi inestimável.

$\mathrm{Na}$ avaliação dos itens referentes ao sistema nervoso, $90 \%$ dos enfermeiros solicitaram retirar os itens da escala de coma de Glasgow e utilizar a escala de Ramsay, uma vez que a maioria dos pacientes de UTI estão entubados. Os juízes justificaram a sugestão afirmando ser esta escala mais aplicável para a avaliação do nível de consciência de pacientes sedados. Foi solicitado reagrupar os aspectos referentes à pressão intracraniana (PIC) nesta categoria.

Nos itens referentes à oxigenação, onde os aspectos de ausculta pulmonar eram discriminados encontrou-se uma concordância pelos especialistas em $100 \%$ dos itens a serem avaliados.
Com relação ao aporte de oxigênio $90 \%$ dos especialistas solicitou a readequação dos itens para melhor organização, pois estes foram considerados extensos. Outra sugestão foi a de acrescentar o item murmúrios vesiculares. Houve ainda a sugestão de $10 \%$ dos juízes de acrescentar ausculta de estridor laríngeo.

No que se refere à necessidade de circulação, $50 \%$ dos juízes solicitou incluir a avaliação do cateter de Swan-Gans, considerando que os dados são essenciais para o controle hemodinâmico do paciente.

Sobre a necessidade de hidratação foi solicitado a associação entre a mesma ao sistema tegumentar além de acrescentar uma escala tipo Likert utilizando cruzes (+) para identificar o nível de hidratação.

Em relação à necessidade de nutrição, 30\% dos juízes solicitaram um maior detalhamento no exame abdominal, com destaque para o número de episódios de evacuações.

Nos itens referentes à necessidade de eliminação, $40 \%$ solicitou destaque para a monitorização da diurese com inclusão de mensuração de 6 em 6 horas (horários de 6, 12, 18 e $24 \mathrm{~h})$.

Para a necessidade de regulação fisiológica, 50 $\%$ dos especialistas sugeriram não relatar todos os exames laboratoriais, já que muitos estão anexos no prontuário do paciente e, segundo os juízes, no instrumento deveria constar os exames onde se identificasse alterações.

Com relação ao item de integridade física, 60 \% dos juízes considerou necessário a inclusão de mais possibilidades na descrição quanto ao aspecto de localização das feridas e seu tratamento.

$\mathrm{Na}$ necessidade de mecânica corporal, $60 \%$ dos especialistas concordaram com os itens propostos para o exame, destacando a importância da inclusão no instrumento de dados referentes à plegias, paresias e parestesias.

Com relação à avaliação das NHB psicossociais, em especial das necessidades de segurança e comunicação, $80 \%$ dos avaliadores concordou com os itens do instrumento, $10 \%$ solicitou a recolocação do item comunicação junto à avaliação neurológica e $10 \%$ sugeriu que se deixasse espaço para as solicitações individuais do pacientes.

Quanto a avaliação da NHB psico-espiritual, grande parte dos juízes (70 \%) concordou com os itens discriminados no instrumento. No entanto, $10 \%$ solicitou acrescentar a previsão de demandas relativas à transfusão sanguínea e outras restrições relacionadas a crenças religiosas e $20 \%$ sugeriu que se deixasse espaço para as solicitações individuais do pacientes.

Os itens foram adequados, acrescentados ou suprimidos quando não se mostraram satisfatórios 
para o atendimento de pacientes críticos em UTI (anexo I).

As sugestões feitas pelos peritos foram acatadas e realizadas readequações sugeridas para os itens referentes aos sinais e sintomas e as terminologias empregadas no instrumento (anexo I).

Destaca-se que $80 \%$ dos avaliadores relatou que a aplicação do instrumento iria facilitar a coleta de dados desde que modificado em aspectos como extensão, ênfase na integridade da pele, alteração na escala de avaliação do nível de consciência. Assim, o instrumento poderia funcionar como guia para o levantamento das necessidades/problemas apresentados pelo paciente. A utilização dos dados a serem coletados poderia favorecer uma visão mais ampla do paciente e aproximaria mais o profissional do paciente, estimulando a interação, identificação de problemas e a individualização do cuidado.

Considerou-se nesta avaliação que o instrumento irá facilitar o registro mais objetivo e claro dos dados a partir das modificações feitas. Neste momento sugere-se a aplicação na prática do instrumento com o objetivo de validar clinicamente os itens que compõe.

Julga-se necessário que o enfermeiro perceba a imperiosidade de documentar suas ações e registrar os sinais e sintomas apresentados pelo paciente que ele observa e os problemas que identifica para subsidiar as ações que deve implementar.

\section{CONCLUSÕES}

A participação dos juízes no processo de avaliação dos itens do instrumento elaborado foi valiosa para sua adequação, pertinência, abrangência, relevância e especificidade.

A criação de um instrumento para a coleta de dados fundamentado na Teoria das NHB de Wanda Horta facilita a aquisição de dados objetivos e subjetivos do paciente. Verificou-se que ele pode facilitar e direcionar a coleta de dados e que serve de guia para a elaboração do plano de cuidados a ser formulado individualmente para os pacientes críticos.

Considera-se que os itens contidos no instrumento proporcionam maior clareza na realização da coleta de dados, podendo facilitar a identificação de diagnósticos de enfermagem que irão subsidiar a formulação de ações no planejamento da assistência, com a descrição de intervenções de enfermagem especificas e individualizadas conforme as NHB identificadas. Destaca-se que uma coleta de dados sistematizada favorece o levantamento de problemas e facilita na determinação do diagnóstico de enfermagem, estabelecimento de intervenções e resultados de enfermagem.

Além disso, a realização deste estudo possibilitou a reflexão sobre a necessidade de estimular e preparar enfermeiros para atuarem de forma sistematizada junto ao paciente e da necessidade de estarem continuamente adquirindo conhecimentos, treinando-se e atualizando-se na utilização do processo de enfermagem, enquanto um método para sistematizar a assistência de enfermagem que prestam a seus pacientes. Ressaltase a importância do processo de enfermagem ser fundamentado em uma teoria, em especial na Teoria das NHB de Horta, uma teórica brasileira que desenvolveu uma teoria adaptada aos nossos costumes e cultura.

Uma limitação do estudo é o processo de validação do instrumento, realizado com relação à aparência e conteúdo, sendo necessária sua validação clínica, para que ele possa contribuir com o cuidado do paciente de forma sistematizada.

O grande desafio é obter instrumentos aplicáveis e construídos com a equipe responsável pelo cuidado e consciente da responsabilidade de implantar uma assistência sistematizada.

\section{REFERÊNCIAS BIBLIOGRÁFICAS}

ALFARO-LEFREVE, R. Aplicação do processo de enfermagem: promoção do cuidado colaborativo. 5 . ed. Porto Alegre: Artmed, 2005.

ALFARO-LEFEVRE, R. Pensamento crítico em enfermagem: um enfoque prático. Porto Alegre: Artes Médicas, 1996.

ANDRIS, D. A. Semiologia: bases para a prática assistencial. Rio de Janeiro: Guanabara Koogan, 2006.

ATKINSON, L. D.; MURRAY, M. E. Fundamentos de enfermagem: introdução ao processo de enfermagem. Rio de Janeiro: Guanabara Koogan, 1998.

BARROS, A. L. B. L de. et al. Anamnese e exame físico: avaliação diagnóstica de enfermagem no adulto. 2. ed. Porto Alegre: Artmed, 2002.

BRASIL, Ministério da Saúde. Conselho Federal de Enfermagem - COFEN. Resolução $n^{\circ}$ 272/2002. Dispõe sobre a Sistematização da Assistência de Enfermagem. Rio de Janeiro, 12 de Julho de 2002. Disponível em < http://www.corenmg.org.br/interna.asp?menu=0\&submenu=5\&prefixos $=272>$. [Acesso em: 11 jan. 2005]

BETEGHELLI, P. et al. Sistematização da Assistência de Eenfermagem em um ambulatório de saúde mental. Revista Eletrônica de Enfermagem, 2005. Disponível em: http://www.fen.ufg.br/revista/revista7 3/original 11.ht m [Acesso em 20 ago. 2006]

CARVALHO, E. C.; GARCIA, T. R. Processo de enfermagem:o raciocínio e julgamento clínico no estabelecimento do diagnóstico de enfermagem In: MONTEIRO, A. B. C.; et al. (Org). FÓRUM MINEIRO DE ENFERMAGEM. 3., 2002, Anais... Uberlândia: UFU, 2002. UFU. 
CARPENITO, L. J. Diagnósticos de enfermagem: aplicação à prática clínica. 6. ed. Porto Alegre: Artmed, 1997.

CHIANCA, T. C. M. et al. Os diagnósticos de enfermagem $e$ as perspectivas da prática de enfermagem para um novo milênio. Revista Mineira de Enfermagem. v.4, n.1/2, p.64-66, 2000.

CIANCIARRULO, T. I. (Org.). Sistema de assistência de enfermagem: evolução e tendências. São Paulo: Ícone, 2001.

DOENGES, M. E.; MOORHOUSE, M. F. Diagnósticos e intervenção em enfermagem. 5. ed. Porto Alegre: Artmed, 1999.

FULY, et al. Planejamento da sistematização da assistência de enfermagem em unidade de terapia semi-intensiva Rio de Janeiro as a support to the development of an electronic patient record. Online Brazilian Journal of Nursing (OBJN - ISSN 16764285) [Online] 2003. Disponível em: <http://www.uff.br/nepae/objn203fulyfreirealmeida.htm $>$ [Acesso em 10 jan. de 2006]

FEITOZA, et al. Traumatismo crânio-encefálico: diagnóstico de enfermagem a vítimas atendidas em UTI. Revista Eletrônica de Enfermagem, [Online] 2004. Disponível em:

<http://www.fen.ufg.br/revista/revista6 2/tce.html>

[Acesso em 15 jul. 2006]

GEORGE, J. B. et al. Teorias da enfermagem. Porto Alegre: Artes Médicas, 2000.

GOMES, G. B. et al. Aplicação do processo de enfermagem a pacientes inconscientes internados na unidade de terapia intensiva de um hospital de Curitiba. Rev. Paul. Enf.. v.7, n.1, p.22-27. 1987.

HORTA, W. A. O processo de enfermagem. São Paulo: EPU/EDUSP, 1979.

HORTA, W. A. Bases of a nursing science. Enf. Novas Dimensões .v.1, n.3, p.105-106. 1975.
HUDAK, C. M.; GALLO, B. M. Cuidados intensivos de enfermagem: uma abordagem holística. 6 . ed. Rio de Janeiro: Guanabara Koogan, 1997.

JARVIS, C. Exame físico e avaliação de saúde. 3. ed. Rio de Janeiro: Guanabara Koogan, 2002.

LOBIONDO-WOOD, G.; HABER, J. Pesquisa em enfermagem: métodos, avaliação crítica e utilização. 4. ed. Rio de Janeiro: Guanabara Koogan, 2001.

LIMA, L. R. de; PEREIRA, S. V. M.; CHIANCA, T. C. M. Diagnósticos de Enfermagem em pacientes póscateterismo cardíaco - Contribuição de Orem. Rev. Brasileira de Enfermagem. v.59, n.3, p.285-290. 2006. Mc CLOSKEY, C. J.; BULECHEK, M. G. Classificação das Intervenções de enfermagem (NIC). $3^{\mathrm{a}}$ ed. Porto Alegre: Artmed, 2004.

PORTO, C. C. Semiologia médica. 4. ed. Rio de Janeiro: Guanabara Koogan, 2001.

POLIT, D. F.; BECK, C. T.; HUNGLER, B. P. Fundamentos de pesquisa em enfermagem: métodos, avaliação e utilização. 5. ed. Porto Alegre: Artmed, 2004.

SPERANDIO, D. J.; ÉVORA, Y. D. M. Planejamento da sistematização da assistência de enfermagem em unidade de terapia intensiva. IX Congresso brasileiro de informática em saúde, São Paulo: [online] 2004. Disponível em:

<http://www.sbis.org.br/cbis9/arquivos/297.doc> [Acesso em 22 mai. 2006]

RISNER, P. B. Diagnostic staments. IN GRIFFITYKENNEY, J. W.; CHRISTENSEN, P. J. Nursing Process: application of theries, frameworks, and models. St Louis: Mosby, 1968.

TIMBY, B. K. Conceitos e habilidades fundamentais no atendimento de enfermagem. 6. ed. São Paulo: Artmed, 2002. 


\section{ANEXOS \\ PARTE I \\ INSTRUMENTO DE COLETAS DE DADOS PARA PACIENTES CRÍTICOS}

\section{Identificação}

Nome: Data de nascimento

Idade___ Sexo: ( ) masculino ( ) feminino

Horário de admissão: Data da admissão:

Diagnóstico: Leito:

Nacionalidade: Naturalidade:

Nível de escolaridade: Estado civil:

Número de filhos:

Tem familiares? Quem? (Descrever)

Procedência:

Informante:

Endereço: Telefone:

\section{Entrevista}

Como e quando começou a sentir problemas?

Doença de base (Início dos sintomas, quais os sintomas)

Algum tratamento já foi iniciado?

Qual a sua ocupação? Antes do problema? E atualmente?

Já se submeteu a alguma cirurgia? Qual?

Fatores de riscos/história pregressa de doença
Cardiopatia congênita ( )
HA ( )
Diabetes ( )
Aterosclerose ( )
Angina ( )
IAM ( )
Obesidade ( )
Insuficiência Renal ( ) Sedentarismo ( )
Uso de contraceptivo oral ( )
Outras informações:

\section{PARTE II}

Nome: Leito: Data: 


\section{1-NHB Psicobiológicas}

Data de admissão

\section{- Percepção}

\begin{tabular}{|l|l|l|l|}
\hline ESCALA DE RAMSAY & Grau & & Grau \\
\hline Acordado, ansioso e/ou inquieto & $\mathbf{1}(\mathbf{~})$ & Dormindo, resposta leve a estímulo tátil ou auditivo & $\mathbf{4}($ ) \\
\hline Cooperativo, orientado e tranquilo & $\mathbf{2 ( ~ )}$ & $\begin{array}{l}\text { Sem Resposta a estímulo auditivo ou tátil, porém com } \\
\text { resposta a dor }\end{array}$ & $\mathbf{5}($ ) \\
\hline Acordado, responde a comandos verbais & $\mathbf{3 ( ~ ) ~}$ & Sem Resposta a estímulo doloroso & $\mathbf{6 ( ~ ) ~}$ \\
\hline
\end{tabular}

\section{SISTEMA NERVOSO}

crise convulsiva ( )

confusão mental ( )

orientado tempo/espaço ( )

usa medicação

\section{CABEÇA/PESCOÇO}

aumento das parótidas ( ) aumentos gl. Tireóide ( ) rigidez de nuca ( )

\section{OUVIDO}

otalgia ( )

hiperacusia ( )

hipoacusia ( )

NARIZ

epistaxe ( )

\section{FARINGE/BOCA}

fenda palatina ( )

dislalia ( )

trismo ( )

\section{- Oxigenação}

Ausculta Pulmonar

\begin{tabular}{|c|c|c|c|c|c|c|c|c|}
\hline $\begin{array}{l}\text { Pontos de } \\
\text { ausculta }\end{array}$ & $\begin{array}{l}\text { Murmúrios } \\
\text { Vesiculares }\end{array}$ & $\begin{array}{l}\text { Sons } \\
\text { Brônquicos }\end{array}$ & $\begin{array}{l}\text { Sons Brônquicos } \\
\text { Vesiculares }\end{array}$ & Creptações & Sibilos & Roncos & Atrito Pleural & $\begin{array}{l}\text { Estridor } \\
\text { Laringeo }\end{array}$ \\
\hline Ápice D & & & & & & & & \\
\hline Base D & & & & & & & & \\
\hline Ápice $\mathrm{E}$ & & & & & & & & \\
\hline Base E & & & & & & & & \\
\hline $\begin{array}{l}\text { Região } \\
\text { Esternal }\end{array}$ & & & & & & & & \\
\hline Brônquio D & & & & & & & & \\
\hline Brônquio E & & & & & & & & \\
\hline Traquéia & & & & & & & & \\
\hline
\end{tabular}

Coloração pele/lábios

$$
\mathrm{SaO} 2
$$

torpor ( )

síncope ( ) sonolência ( )

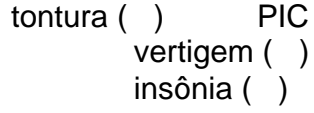

tontura ( ) PIC vertigem ( ) insônia ( )

prurido ( )

otorréia ( )

otorragia

coriza ( )

leucoplasia/língua ( ) inflamação ( ) lábio leporino ( ) disfonia ( ) disfagia ( ) halitose ( ) traqueostomia ( )

surdez ( )

$$
\text { secreção ( ) }
$$

rinorréia ( ) obstrução ( )
OLHOS secreção ( )

nistagmo ( ) acuidade visual dimunuida ( ) fotofobia ( ) diplopia ( ) escotoma ( ) edema palpebral ( )

zumbido ( )

Enchimentos ungueais

$\mathrm{SpO} 2$

Aporte de oxigenação:

ar ambiente ( )

VMI ( )

algia torácica( )

tosse produtiva ( )

cianose ( )

Secreção traqueal:

tiragem intercostal ( )

- Circulação

\section{SISTEMA CARDIOCIRCULATÓRIO}

Ritmo cardíaco: Sinusal ( ) Arritmico ( )

Dor no peito (localização):

O que melhora e o que piora a dor?

bradicardia ( )

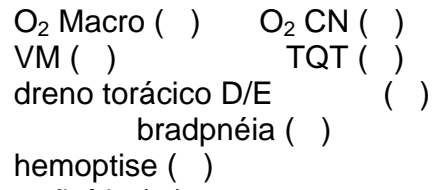

$\mathrm{O}_{2} \operatorname{Macro}()^{\prime} \quad \mathrm{O}_{2} \mathrm{CN}(\quad)$ VM ( ) TQT ( )

dreno torácico D/E ( ) bradpnéia ( )

hemoptise ( )

fluída ( )

cornagem ( )

\section{$\mathrm{O}_{2}$ Venturi ( ) $\mathrm{O}_{2}$ Masc. Simples ( )}

soluço ( ) apnéia ( ) tosse seca ( ) eupnéia ( ) expectoração cor/aspecto ( ) batimento asa nasal ( )

espessa( ) sanguinolenta( ) purulenta( ) precordialgia ( ) taquicardia ( ) Duração Intensidade

pulso arritmico (

Swan-Gans: PAD pulso rítmico ( ) $\mathrm{mmHg} \quad \mathrm{PVD} \quad \mathrm{mmHg}$

PAP $\mathrm{mmHg}$

PCCP $\mathrm{mmHa}$ 
SvO2

DC

RVS

RVP

Ausculta cardíaca

\begin{tabular}{|c|c|c|c|c|c|c|c|}
\hline $\begin{array}{l}\text { Focos de } \\
\text { ausculta }\end{array}$ & $\begin{array}{l}1^{\mathrm{a}} \\
\text { Bulha }\end{array}$ & $\begin{array}{l}2^{a} \\
\text { Bulha }\end{array}$ & $\begin{array}{r}\text { Som de } \\
\text { Galope }\end{array}$ & $\begin{array}{l}\text { Atrito } \\
\text { Pericárdico }\end{array}$ & Sopros & $\begin{array}{c}\text { Sinais } \\
\text { e Sintomas }\end{array}$ & $\begin{array}{c}\text { Sinais } \\
\text { e Sintomas }\end{array}$ \\
\hline Foco Aórtico & & & & & & Palpitações & $\begin{array}{l}\text { Ingurgitamento } \\
\text { da jugular }\end{array}$ \\
\hline $\begin{array}{l}\text { Foco } \\
\text { Pulmonar }\end{array}$ & & & & & & Síncope & $\begin{array}{l}\text { Rede venosa } \\
\text { visível no tórax }\end{array}$ \\
\hline Ictus cordis) & & & & & & $\begin{array}{l}\text { Intolerância a } \\
\text { atividade }\end{array}$ & Edema (local) \\
\hline $\begin{array}{l}\text { Foco } \\
\text { Tricúspede }\end{array}$ & & & & & & Frêmitos & \\
\hline
\end{tabular}

- Regulação fisiológica

Sinais vitais

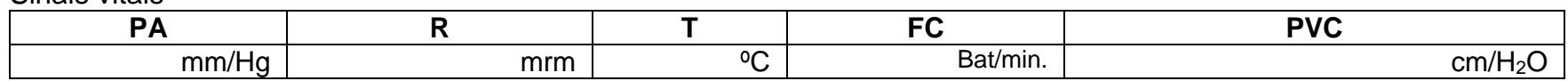

Exames Laboratoriais:

$\bullet \quad$ Hidratação
Hidratação: Oral ( ) Soroterapia ( )
Turgor cutâneo

\section{- Nutrição}

SISTEMA GASTRO INSTESTINAL

Tipo dieta:

Aceitação:

suspensa ( ) Oral ( )

satisfatória ( ) anorexia( )

$\mathrm{NPT} \quad \mathrm{ml} / \mathrm{h} \quad \mathrm{NE}$ $\mathrm{ml} / \mathrm{h}$

Tipo sonda:

SNG ( ) SNE ( )

hiporexia ( )

Eliminações: vômitos ( ) estase ( )

RHA: ausente ( ) presente ( )

Abdome: distendido ( )

globoso ( )

SNE pilórica ( ) gastrostomia ( ) jejunostomia ( )

diminuído ( )

tenso ( ) flacido ( ) plano ( )

\section{- Eliminação}

Freqüência eliminação intestinal

Colostomia ( )

enterorragia ( )

\section{SISTEMA GENITO URINÁRIO}

diurese normal ( ) anúria ( )

polaciúria ( )

\section{disúria ( )}

hidrocele ( )

retenção urinária sonda alívio ( )

SVD: Fechado ( ) Aberto ( ) $6 \mathrm{~h}$

\section{- Integridade física}

\section{PELE}

eritema ( )

hematoma ( )

pápula ( )

escoriação ( ) desidratada ( ) prurido ( )

úlcera pressão- grau I ( )grau II ( )

Tratamento/curativo

ASPECTOS HIGIENE

escabiose ( ) pediculose ( )

condições de higiene: satisfatória ( )

\section{- Mecânica corporal}

\section{ÓSTEO/ARTICULAR}

artralgia ( )

atrofia muscular ( )

cãimbra ( )

\section{2-NHB Psicossociais}

$$
\text { - Segurança }
$$

\section{espasmo muscular ( ) cervicalgia ( )} fraqueza muscular ( ) lombalgia ( ) lombocitalgia ( )

\section{ASPECTOS EMOCIONAIS}

$\begin{array}{llll}\text { Apático ( ) } & \text { depressivo ( ) angústia ( ) } & \text { calmo ( ) } & \text { Comunicação verbal ( ) } \\ \text { agressivo ( ) colaborativo ( ) ansioso ( ) } & \text { comunicativo ( ) } & \text { Comunicação não verbal ( ) } \\ \text { Solicitação do paciente } & \end{array}$

Solicitação do paciente
hematúria ( )
oligúria ( )
poliúria ( ) incontinência urinária ( ) urina turva ( ) cistostomia ( ) leucorréia( ) menorragia( ) metrorragia( ) Diálise ( ) $\mathrm{ml} 12 \mathrm{~h}$ $\mathrm{ml} 18 \mathrm{~h}$ $\mathrm{ml} 24 \mathrm{~h}$ $\mathrm{ml}$

fissura ( ) herpes zoster ( ) bolha ( ) hidratada ( ) crosta ( ) Incisão cirúrgica ( ) petéquia ( ) grau III ( ) grau IV $($ )

\section{3-NBH Psico-espiritual}

Religião Solicitação do paciente 